\title{
Aportes de las teorías sociológicas a la discusión de la ontología. Los casos de Luhmann, Habermas y Latour
}

\section{(Sociological theories contributions to the ontological question. The cases of Luhmann, Habermas y Latour)}

\author{
Sergio Pignuoli Ocampo
}

Recibido: 30 de septiembre de 2014

Aceptado: 7 de octubre de 2015

\section{Resumen}

En este trabajo se discuten los aportes de la teoría sociológica contemporánea al debate filosófico y científico de la ontología, para ello son cotejados los componentes ontológicos de la Teoría General de Sistemas Sociales de Niklas Luhmann, 1la Teoría de la Acción Comunicativa de Jürgen Habermas y la Actor-Network Theory de Bruno Latour.

Palabras clave: Ontología, Luhmann, Latour y Habermas.

\begin{abstract}
In this paper are discussed the contributions of the contemporary sociological theory to the philosophical and scientical question of Ontology, for this purpose are compared the ontological assumptions elaborated for the General Social Systems Theory by Niklas Luhmann, Communicative Action Theory by Jürgen Habermas and Actor-Network Theory by Bruno Latour.
\end{abstract}

Keywords: Ontology, Luhmann, Latour y Habermas. 


\section{Presentación}

Como es sabido la discusión de la ontología y, más precisamente, la discusión de la fundamentación metafísica de ella es uno de los temas más atractivos y cautivantes de la reflexión teórica del siglo veinte. En el campo de la filosofía esta discusión ha merecido amplios y profundos aportes. Pensadores tan diversos como Heidegger (2006), Derrida (1967) o Rorty (1979), entre otros destacados, han considerado las bases causales mismas de la metafísica y han llevado sus críticas y cuestionamientos de ella hasta umbrales insospechados de la reflexión ontológica. Aunque alcanzó picos notables en el terreno filosófico, la discusión no se agotó en él. Ella recibió aportes sustantivos desde el campo de las ciencias empíricas. De este modo la "interpretación ontológica" de los avances en el conocimiento científico se constituyó en una problemática de investigación adicional para las mismas disciplinas, según se ha observado ya en campos tan diversos como la lingüística (Rosa 1991), la biología celular (Maturana y Varela 2003), la física cuántica (d'Espagnat 2006) y la cibernética (Hagner y Hörl 2008).

Dentro de ese contexto es oportuno someter a consideración el papel desempeñado por las ciencias sociales en la discusión general de la ontología y la metafísica. En cuanto a la contribución de ellas al campo de la ontología destaca el rol jugado por la sociología en la postulación y el desarrollo del programa de la ontología social. Georg Simmel, Alfred Schütz y Anthony Giddens fueron los tres sociólogos que abonaron con mayor esfuerzo e innovación las bases teóricas de aquél. Simmel (1908) bajo las premisas del neokantismo rickertiano elaboró una apriorística social, que establece de manera innovadora los fundamentos de una ontología fundada en lo social y sus formas. Schütz (2003), el teórico más importante de este programa, desarrolló desde la fenomenología poshusserliana una ontología del mundo de la vida enfocada sobre las preconstrucciones significativas del mundo y su relevancia para la interacción mundana de los hombres. Giddens (1984) en el marco del giro lingüístico de las ciencias sociales hizo un desarrollo ulterior de la ontología schütziana y se propuso deslindar la relación práctica contingente entre agentes reflexivos y estructuras significativas.

Estos antecedentes, entre otros, convierten al programa de la ontología social en la contribución más importante de las ciencias sociales al campo de la ontología. Si bien es cierto que es difícil encontrar fuera de ella otro programa de investigación social que valore de igual manera la cuestión ontológica y que elabore en torno a ella una heurística positiva para su progreso programático, no es menos cierto que los principales programas sociológicos inaugurados desde las últimas décadas del siglo XIX hasta el periodo de entreguerras del siglo XX dialogaron e hicieron propios los tópicos de la investigación ontológica y metafísica. Esto se puede observar, entre otros notables ejemplos, en los trabajos de Gabriel Tarde (1893), Ferdinand 
Tönnies (1887), George Herbert Mead (1931) e incluso en filósofos marxistas que seguían de cerca la evolución de la sociología como György Lukács (1923). Esta consideración positiva de las cuestiones ontológicas y metafísicas fue modificada sustancialmente en las escenas europea y estadounidense de la posguerra temprana y tardía. Ponemos el acento sobre este periodo porque en él se produjo la expansión de la investigación social aplicada. Ella desató abiertamente la disputa sobre el papel y la función de la teoría en las ciencias sociales y reclamó que las restricciones metodológicas que enarbolaba recibieran la dignidad de criterios teóricos generales de la sociología. El desprecio de la investigación social aplicada por la discusión conceptual en general, a la que consideró metafísica irrelevante para el estudio de objetos sociales, desplazó la materia ontológica de las agendas de investigación de los programas de mayor impacto. En ese marco surgió una opinión, según la cual, las ciencias sociales se habrían mantenido al margen y no habrían realizado contribuciones importantes a la discusión de la ontología y de la metafísica a lo largo del pasado siglo, ya sea porque se considerara que el debate en torno a la ontología y la metafísica era ajeno tanto a su perspectiva y materia como a su estilo de construcción de objetos y problemas (Schaeffer 2009), ya sea porque se estimara que la sociología atravesaba una fase de retraso relativo respecto de los aportes filosóficos, epistemológicos o de otras disciplinas científicas (Buckley 1973).

Esa opinión se esparció rápidamente y sus ecos llegan hasta nuestros días. Ella da cuenta, de un modo exagerado y sesgado, de una situación de hecho: una rama importante de las ciencias sociales, la investigación social aplicada, se desentendió de y llamó a erradicar los debates ontológicos en la sociología. Sin embargo, tal opinión no agota, ni mucho menos, las diversas y heterogéneas relaciones de las ciencias sociales con los referidos debates. En este trabajo la rebatiremos, ya que nos proponemos analizar los aportes a la discusión de la ontología realizados desde la sociología, en especial desde la teoría sociológica reciente. Lejos de ser refractaria hacia aquélla, la teoría sociológica de posguerra ha mostrado un gran interés por retomar e incluso por ofrecer elementos propiamente disciplinarios a la cuestión. Desde los usos dados por Talcott Parsons (1953) a la biología de Cannon y Henderson hasta la complementación sugerida por Pierre Bourdieu (1985) entre sociología y morfología estructural, observamos que las innovaciones teóricas de la sociología han encontrado en el diálogo transdisciplinario una clave para problematizar en y desde las ciencias sociales en general y la sociología en particular las premisas metafísico-ontológicas de sus principales programas de investigación. La discusión sobre la fundamentación metafísica de la ontología no quedó al margen de estos esfuerzos de los programas interesados en la teoría de la disciplina por renovar la concepción de la unidad de análisis y de la unidad del campo de la sociología. En este trabajo analizaremos puntualmente los componentes ontológicos de tres programas de teoría sociológica actual: la Teoría General de Sistemas Sociales (TGSS) de Niklas Luhmann, la Actor-Network Theory (ANT) de Bruno Latour y la 
Teoría de la Acción Comunicativa (TAC) de Jürgen Habermas. Esta terna no agota el conjunto de programas sociológicos que se hizo cargo de que la discusión de la fundamentación metafísica de la ontología contiene problemas que alcanzan la base causal misma de la disciplina. Baste invocar los nombres de Jon Elster (1978) y Margaret Archer (1988), quienes también han tratado esta cuestión con toda la seriedad del caso. Sin embargo la TGSS, la ANT y la TAC son los tres programas que han elaborado esta discusión con mayor radicalidad y profundidad. Los tres programas asumen que, sin una adecuada apreciación y tratamiento de ella, hasta la unidad de análisis de la sociología quedaría indeterminada. Por esta razón observamos que ellos convirtieron el problema de la metafísica en el problema ontológico fundamental de sus programas, un problema que debían resolver adecuadamente para reformular de manera satisfactoria los componentes ontológicos de sus respectivos programas. La radicalidad de estas pretensiones fue, además, asociada por los tres autores con la autoproclamación de sus propuestas como "cambios de paradigma" en la sociología. Estos elementos son el factor común que nos permite aunarlos y estudiarlos de manera comparada en relación con el tema de referencia.

En este punto nos apresuramos a introducir una importante aclaración: no pretendemos contrariar las explícitas críticas de estos autores contra la "metafísica" y la "ontología". Más bien nos interesa establecer las premisas teórico-metodológicas para comparar sus conceptos primordiales respecto de la estructuración de lo real, en cuyo marco critican la "ontología". Nos valemos para ello de una distinción de Schaeffer (2009), quien deslindó dos conceptos de ontología: el primero concierne a la adopción de premisas sobre la estructuración de lo real, tiene un sentido amplio y general; el segundo, en cambio, es cultural e históricamente determinado, pertenece a la definición de la estructuración de lo real predominante en la tradición del pensamiento filosófico europeo occidental, que se basa en las premisas del dualismo ontológico, la centralidad de las categorías de sustancia y esencia, el gnoseocentrismo y el antinaturalismo. Schaeffer encuadró el segundo concepto dentro del primero y afirmó que se trata de un caso histórico y específico de elaboración conceptual respecto de la estructuración de lo real. ${ }^{1}$ En este sentido la crítica del "proyecto ontológico de Occidente", incluso las críticas "radicales", objetan el segundo concepto de ontología, es decir, objetan una de las ontologías posibles. No se sigue necesariamente de ello una crítica o una renuncia al primer concepto de ontología, esto es, a la ontología en general. Por consiguiente, una vez cuestionado el segundo concepto de ontología, la continuidad del planteo crítico enfrenta una bifurcación entre dos modos de argumentación ontológica, o bien, si sigue el primer modo, propone una fundación basada sobre otras premisas que permitan replantear la

\footnotetext{
1 Si bien el planteo de Schaeffer es oscilante al respecto, el autor estableció, en cualquier caso, una relación de premisa mayor y premisa menor entre los términos y tomamos de entre sus diversos planteos aquél cuya terminología mejor nos asistía en la definición del componente ontológico.
} 
estructuración de lo real de manera alternativa, o bien, si sigue el segundo modo, extiende el cuestionamiento hacia el primer concepto de ontología y realiza una segunda crítica respecto de toda ontología. Únicamente esta última modalidad renuncia por fuerza a toda elaboración conceptual referente a la estructuración de lo real. No conocemos ningún programa sociológico ni científico que la adopte. Luhmann, Latour y Habermas asumen con firmeza la primera modalidad: critican "radicalmente la ontología" y/o la "metafísica de Occidente" a partir de sus propias concepciones de estructuración de lo real. Por consiguiente, sin negar sus "críticas radicales contra la ontología", reconstruimos y cotejamos desde una perspectiva sistemática los componentes ontológicos de la TGSS, la ANT y la TAC. ${ }^{2}$

Con este planteo se despeja el panorama y observamos que nuestros programas no sólo elaboran conceptualmente componentes ontológicos, sino que estos son generales y meticulosos. La TGSS los elabora en torno a la complejidad y la emergencia de sistemas, la ANT lo hace en torno a la heterogeneidad relacional de los actantes y la TAC hace lo propio sobre la constitución lingüística del acceso al mundo. El abordaje exhaustivo de estas ontologías excede nuestros objetivos y es innecesario a tales efectos. Para darles un tratamiento comparado acotado y adecuado, los desagregramos. Mascareño (2008, p. 221) identificó tres dimensiones ontológicas básicas: 1) tiempo y espacio, 2) composición de entidades y 3) relaciones causales entre ellas.

La exposición sigue este plan: para ofrecer una imagen global presentamos por separado la complejidad y la emergencia de la TGSS (2), la heterogeneidad, las fuerzas y la relacionalidad de la ANT (3) y la constitución lingüística de la apertura al mundo de la TAC (4), luego los cotejamos (5) y extraemos las conclusiones (6).

\section{TGSS: complejidad y emergencia de sistemas ${ }^{3}$}

Luhmann consideró la "tradición ontológica" como una semántica histórica vetero-europea. En contra de ella elaboró un fundamento operativo capaz de

\footnotetext{
2 No somos los primeros en afirmar que además de ser críticos "radicales" de la "metafísica ontológica", Luhmann, Latour y Habermas elaboran sus propias ontologías. Al respecto de Luhmann están los trabajos de Clam (2000) Rustemeyer (2007) y Rasch (2012); al respecto de Latour, están los trabajos de Harman (2009); y al respecto de Habermas, los escritos de Lafont (1993), Fabra (2008). Vandenberghe (2007) ofrece una de las pocas ontologías que integra, entre otros, a nuestros tres autores. Para una visión general respecto de la "ontología científica" remito a Bunge (2004).

3 A fin de familiarizar al lector con los tres programas que estudiaremos en los apartados en que expongamos sus concepciones ontológicas presentaremos abreviadamente sus programas. En cuanto a la TGSS, la producción de Luhmann abarca más de tres décadas, desde su proclamación con la publicación de Funktionale Methode und Systemtheorie ("Método funcional y teoría de sistemas") en 1964, hasta su último gran producto Die Gesellschaft der Gesellschaft ("La sociedad de la sociedad") de 1997. El proyecto luhmanniano se declaró, desde un primer momento, interesado en establecer la
} 
"desontologizar la realidad" (2009, pp. 35-6). Luhmann rechazó los principios fuertes de la "ontología vetero-europea": identidad y necesidad, así como sus fundamentos primordiales: sustancia, esencia y trascendencia, y los sustituyó con principios de la teoría de la complejidad y de la emergencia de sistemas.

El concepto de complejidad es el más comprehensivo de la TGSS. ${ }^{4}$ En cuanto referida a sistemas, Luhmann la definió como la imposibilidad de un sistema de mantener actualizados simultáneamente la totalidad de sus elementos y relaciones. ${ }^{5}$ Un sistema complejo carece de tal capacidad porque su organización interna ha traspasado un umbral a partir del cual sólo puede actualizar los estados de sus elementos y relaciones mediante selección, es decir, es un sistema forzado a seleccionar (Selektionzwang). ${ }^{6}$ Por esta razón, la selección es central para el concepto y en torno a él se articulan las principales propiedades de la complejidad, a saber:

- Diferencial de complejidad (Komplexitätgefalle): para un sistema complejo seleccionar no significa tomar ("importar") elementos del mundo y emplearlos como propios, sino diferenciar un esquema distribuido de posibilidades e imposibilidades de actualización de estados. Este esquema, por un lado, excede la capacidad limitada de actualización del sistema, de manera que éste está forzado a distribuir

sociología como ciencia de los sistemas sociales. En su evolución distinguimos tres fases. La primera (1964-1984) se caracteriza por la asunción de la teoría de la acción y la teoría funcional cibernética de sistemas; el punto de inflexión es la publicación de Soziale Systeme ("Sistemas sociales"). La segunda (1984-1990) se caracteriza por la asunción de las teorías de la comunicación y de sistemas autorreferenciales autopoiéticos clausurados en la operación. La tercera fase (1990-1997), se caracteriza por un mayor énfasis en la epistemología y la metodología de la teoría de la forma; el punto de inflexión es la publicación de Die Wissenschaft der Gesellschaft ("La ciencia de la sociedad"). Aquí enfocaremos la segunda fase, en ese momento Luhmann imprimió a la TGSS giros autopoiético, comunicativo y emergentista, de manera que diferenció su fundamento operativo respecto de "la teoría de la acción", del "funcionalismo" y de la "amalgama de teorías" y les contrapuso un giro emergentista, pero también respecto de la tradición sistémica de la sociología a la que contrapuso los giros autopoiético y comunicativo. En ese marco, Luhmann diseñó un programa sistémico, con pretensiones de universalidad y autoimplicación en el ámbito del objeto. Elaboró conceptualmente la comunicación como fundamento operativo novedoso y en torno a ella desarrolló un componente ontológico basado en las teorías de la complejidad y de la emergencia, un componente epistemológico orientado por el constructivismo operativo, y un componente metodológico regido por la observación de segundo orden.

${ }^{4}$ Luhmann indicó que su definición de complejidad concierne tanto a sistemas como a no-sistemas. Respecto de los últimos, su principal uso fue imputarle al mundo (Welt) el atributo de complejo. En el mundo la actualización de elementos y relaciones es equiprobable, es decir, cualquier actualización entre cualquier elemento y cualquier relación es posible e igualmente probable (atributo de entropía), pero es imposible actualizar todo simultáneamente (atributo de complejidad). Para la TGSS el mundo no es un sistema, ya que, si lo fuera, debería distinguirse de un entorno, lo que contradiría el concepto. 5 Sistemas Sociales ofrece dos definiciones de complejidad, la aludida se basa en la diferencia elemento/relación; la segunda la define como "la medida de la indeterminación de un sistema" (1984, pp. 501) y se basa en la diferencia información/indeterminación.

6 El concepto de umbral es caro al campo de la complejidad. J. von Neumann (1966) lo empleó para introducir, por primera vez, el concepto de complicación, y luego el de complejidad. 
las posibilidades en selectivamente probables e improbables, conformando un horizonte interno de selección, y por otro lado, más importante, constituye un orden de complejidad reducida (i.e. no equiprobable) dentro de la complejidad del mundo (i.e. equiprobable). En consecuencia, un sistema complejo establece entre él y su entorno un diferencial de complejidad, que sólo él puede regular. Por esta razón la TGSS concibe el diferencial de complejidad en el seno de un continuo material: los sistemas operan dentro de un único mundo, lo transforman con sus propias operaciones sin trascenderlo.

- Temporalización: la selección posee una estructura temporal de hierro: ninguna selección es perpetua, todas son acontecimientos irreversibles en el tiempo y tienen una duración. Mientras dura, toda selección abre la posibilidad de enlace y presiona sobre la selectividad del sistema, de manera tal que, o bien es sucedida por una nueva selección, o bien el sistema colapsa al ser incapaz de actualizarse, en cuyo caso el diferencial de complejidad se desorganiza y se extingue. Por esta razón la complejidad es temporalizada.

- Contingencia del orden complejo: ninguna selección es necesaria ni imposible, sólo forzada (1984, p. 47). Por tanto cada selección de un orden complejo supone otras determinaciones posibles, así toda selección pudo, puede y podrá ser de otro modo. Este carácter establece que todo orden complejo es inexorablemente contingente.

- Una operación es un elemento complejo: dado que seleccionar no es simplificar, los elementos últimos (Letztelemente) de un orden complejo no son unidades simples (átomos, individuos), sino elementos complejos, llamados operaciones. Una operación es una unidad compleja que diferencia al sistema dentro del mundo, pues lo cierra operativamente ante el entorno y las operaciones de otros sistemas que, por definición, son incapaces de operar en él.

- Seleccionar reduce y aumenta complejidad, no la simplifica: un sistema complejo es tan incapaz de alcanzar selectivamente elementos y/o relaciones simples como de simplificar(se) la complejidad. La selección más bien reduce complejidad al momento de actualizarse como tal, dicha reducción aumenta la complejidad para selecciones (reducciones) futuras en el orden complejo. Toda selección actualiza como unidad determinados elementos y relaciones, mientras mantiene otros como posibles.

El fundamento ontológico de la diferenciación y el estar-en-el-mundo de los sistemas complejos es un tema que nos lleva al concepto de emergencia de sistemas autorrefenciales. Para la TGSS, un orden de complejidad reducida es un orden emergente. Emergencia significa aparición de entidades y propiedades cualitativamente novedosas dentro del mundo, cuyas propiedades no se derivan ni están presentes en sus precursores ni en sus condiciones de posibilidad. Esto significa que las propiedades emergentes suponen al mundo, no lo crean (nada emerge de la 
nada), ${ }^{7}$ y suponen también determinadas condiciones de posibilidad y precursores, pero no se derivan causalmente de ellos. Ello destaca que ni las condiciones de posibilidad ni los precursores son razón suficiente de la emergencia. Así leemos: "Emergencia no es simplemente acumulación de complejidad, sino interrupción y nuevo comienzo de la construcción de complejidad" (1984, p. 44).

La TGSS adoptó el enfoque emergentista de la constitución múltiple (multiple constitution). ${ }^{8}$ Para este enfoque "la autorreferencia en el nivel de los procesos básicos sólo es posible cuando existen por lo menos dos procesadores de información que se pueden referir uno al otro, y por medio de uno y otro, a ellos mismos. De modo que la autorreferencia presupone una infraestructura correspondientemente discontinua" (1984, p. 191). Este enfoque enfatiza la no-linealidad de la emergencia respecto de la concurrencia múltiple de base: un orden emergente no selecciona ni combina elementos de los sistemas concurrentes, ellos no participan del orden cuya emergencia posibilitan, están en el entorno de él. Por todas estas razones la emergencia es el basamento que permite a la TGSS afirmar que "hay sistemas", es central para este concepto y en torno a él se articulan sus propiedades principales, a saber:

- Conjunción de causalidad y novedad: un orden emergente está causalmente abierto y cumple diversas legalidades (termodinámicas, físicas, químicas, bioquímicas, biológicas, psíquicas). Por ello es completamente dependiente del mundo en materia de condiciones de posibilidad y precursores, sin embargo, al mismo tiempo, introduce en ese mundo una diferencia inexistente en las condiciones y precursores de los que depende. Ello lo vuelve completamente novedoso. Por esta razón la novedad es considerada como no-lineal respecto de la cobertura nomológica: no es causada ni se deduce de ella. 9

- Irreductibilidad: un orden de constitución múltiple es un orden emergente, por ello sus propiedades no se reducen a las legalidades que cumple ni a los sistemas concurrentes que supone. Las propiedades emergentes son de un nivel superior

\footnotetext{
${ }^{7}$ La plena presunción del mundo como condición necesaria de toda emergencia rebate dos objeciones típicas que ha recibido el emergentismo, primero, es rebatida la acusación de creacionismo, pues un orden emergente no crea al mundo, lo supone; segundo, es rebatida la acusación de misticisimo, pues asume que un orden emergente no supone la nada, sino una gran cantidad y variedad de condiciones mundanas, que la vuelven improbable. La emergencia reformula así el principio de ex nihilo nihil fit ("de la nada nada llega a ser"), contrastándolo a los principios de creatio ex nihilo ("creación de la nada") y de ex nihilo fit ens creatum ("el ente creado llega a ser a partir de la nada") de la teología cristiana.

8 No es sencillo encuadrar la posición emergentista de Luhmann. Solo podemos señalar que el enfoque de la constitución múltiple guarda algunas similitudes con el modelo de "realizabilidad múltiple" (multiple realizability), según el cual, el macronivel depende causalmente de la interacción entre los precursores del micronivel; no obstante, la posición de Luhmann sobre la irreductibilidad causal impide identificarlo plenamente con este modelo, proveniente en sus orígenes de la neurociencia.

${ }^{9}$ Según Luhmann el único caso en que el entorno actúa causalmente en el sistema es la destrucción.
} 
(high order) y sus reglas no se derivan lógicamente ni se reducen a las reglas del sustrato o nivel inferior (low order).

- Diferencia sistema/entorno y diferencia sistema/sistema: un sistema emergente sólo puede operar y reproducirse enlazando nuevas operaciones a partir de operaciones anteriores. Su unidad no es un supuesto, debe operarla para sí mismo (für sich selbst), con independencia de la observación de otros sistemas. Las operaciones, y sólo ellas, mantienen el diferencial de complejidad. Un sistema cerrado en la operación está cerrado al entorno y a los sistemas que operan en él. Sin embargo, no es un sistema aislado, pues en otros planos está abierto al entorno, tanto al nivel condicional, observacional como cognitivo. Ello impide que un sistema cerrado operativamente se independice del entorno y de las dinámicas heterogéneas que en él acontecen. Luhmann (1984, p. 35) afirma así que un sistema complejo está estructuralmente orientado al entorno. ${ }^{10}$ Un sistema complejo no es por tanto una identidad que persevera, sino una diferencia sistema/entorno. Las relaciones intersistémicas suponen tal diferencia y toman la forma de una diferencia sistema/sistema. Entre ellos no hay intercambios, sino una co-orientación entre sistemas situados recíprocamente en el entorno

\section{ANT: heterogeneidad relacional del actante ${ }^{11}$}

Latour basó el componente ontológico de su programa en el concepto de actante y su sistema de categorías (fuerzas, materias y eventos). Sobre esta base la ANT,

\footnotetext{
10 La diferencia sistema/entorno hace del entorno un fundamento, no un residuo del sistema, está en su "base" (1984, p. 242), lo refiere y lo incorpora en su unidad de manera permanente. En virtud de esta orientación hacia un entorno variable, un sistema que opera de manera cerrada, es incapaz de fundamentarse a sí mismo, y, por esta razón, no es subiectus, no es fundamento de sí, por esta razón la diferencia sistema/entorno es la distinción fundamental del componente ontológico luhmanniano.

11 En cuanto a la ANT, la producción de Latour se inició hace más de tres décadas, su primera publicación destacada se remonta a 1979, el trabajo con Steve Woolgar: Laboratory Life, y llega hasta nuestros días, con la reciente edición de Enquête sur les modes d'existence ("Encuesta sobre los modos de existencia") de 2013. Tempranamente el programa latouriano se declaró partidario de convertir la ciencia social en ciencia de las asociaciones. En él distinguimos tres fases: la primera (1977-1987) se caracteriza por el delineamiento de una sociología de la traducción orientada al área de la ciencia y la tecnología, dialogó críticamente con el constructivismo social, o "Programa Fuerte", de Bloor; el punto de inflexión es la publicación de Science in Action en 1987. El segundo momento (1987-1999) se caracteriza por la adopción del principio de simetría generalizada y por el paso del constructivismo social crítico a un constructivismo. Este doble movimiento recibirá la denominación de Actor-Network Theory; el punto de inflexión es la ruptura definitiva de la ANT con la Escuela de Edimburgo, tras la polémica Latour-Bloor. El tercer momento (1999, hasta la actualidad) se distingue por la articulación del programa constructivista con el programa simétrico, y por la estabilización del sistema de categorías relativo a las redes y una mayor atención a la noción de trayectoria. Aquí enfocaremos las fases segunda y tercera, en ese momento Latour dotó a la ANT de un giro hacia la simetría generalizada, así
} 
por un lado, declaró ontológicamente impertinentes las categorías de sustancia, esencia y sujeto, y rechazo las metafísicas tradicionales basadas en ellas (el monismo sustancialista, el esencialismo y el elementalismo simples y/o el dualismo subjetual, sea de sustancia, propiedad o materia), por otro lado, pregonó la ineludibilidad de la metafísica para definir los objetos científicos, incluido el sociológico (2005, p. 51 n.51). A tal efecto Latour elaboró una ontología de la heterogeneidad y la traducción, de la irreductibilidad y la insustancialidad de lo único que existe y deja de existir dentro del mundo: los actantes. Latour defendió la fundamentación ontológicamente débil de su metafísica por su especial pertinencia para describirlos.

Según la ontología de la ANT el mundo consiste de fuerzas, materias y eventos. Las fuerzas son pruebas (épreuves) y todo aquello que las resista es real (1984, p. 177). ${ }^{12}$ Las materias son concreciones reales dotadas de propiedades actanciales. ${ }^{13}$ Los eventos son la puesta en acto, la performación, de una relación específica entre determinadas fuerzas y materias, acontecen en un momento y un lugar determinados. 14

Los actantes construyen realidad dentro (y nunca fuera) de ese mundo. Un actante es una unidad eventual de fuerzas y materias, acontecida en un momento y un lugar determinados. ${ }^{15} \mathrm{El}$ actante, por tanto, construye realidad en relación con tales componentes del mundo. En relación con las fuerzas, el actante requiere de ellas para mantener unida la heterogeneidad que lo constituye de momento a momento e imprimirle una dirección. Por ello los actantes son tanto ensamblados

diferenció su fundamento operativo respecto de "la sociología de lo social", de la "teoría de la acción" y de las "fórmulas de compromiso micro-macro" y les contrapuso la asociación performativa; se diferenció además del programa constructivista, al que contrapuso un giro hacia la simetría generalizada. En ese marco, Latour delineó un programa constructivista, con pretensión de simetría generalizada y relatividad de la investigación. Concibió la asociación como fundamento operativo novedoso y en torno a ella desarrolló un componente ontológico orientado hacia la heterogeneidad del actante, un componente epistemológico basado en el constructivismo simétrico, y un componente metodológico basado en la cartografía de controversias (una presentación más detallada de estos lineamientos se puede encontrar en Pignuoli Ocampo 2015).

12 Latour relativizó la pretensión de exclusividad de la física respecto de la definición de fuerza. Optó, en cambio, por la semiótica actancial de Greimas y la narratología de Bal. Høstaker (2005) agregó que el autor trabajó con la etnometodología del actor.

13 Sobre esta base Latour acusó a las diversas tradiciones materialistas y al trascendentalismo de incurrir en un idealismo materialista y las acusó de ser incapaces de tratar con materias que no son materiales y con máquinas que no son mecánicas.

14 Latour sigue explícitamente el concepto de evento de Whitehead, así un evento es la plena concreción del ente, que por ende no consiste de una sustancia fija y de atributos accidentales, sino que todo el tiempo (que es la temporalidad del evento) es pleno, por tanto atributos y sustancia no se distinguen. Sobre esta base Harman (2009, p. 30) lo señaló como el "anti-Bergson", pues de acuerdo con su eventualidad el actante carece de durée.

15 Harman (2009, pp. 158, 162ss) señaló que Latour apoyó la definición de actante sobre un concepto de "emergencia funcional". Este concepto se ciñe a los modelos de upward causation, pues el principio de irreductibilidad del actantes rechaza in toto los modelos de downward causation. 
de fuerzas $(1984$, p. 223) como líneas de fuerza $(1984$, p. 192) y son capaces de poner a prueba y modificar la correlación y dirección de las fuerzas que performan. En relación con las materias, un actante es un híbrido material capaz de mediar la materia. Finalmente, en relación con los eventos, un actante es definido como un evento en trayecto. Es un evento por cuanto pone en acto todas sus propiedades actanciales en un punto dado del tiempo. Es un trayecto, pues la movilización del actante unifica la sucesión de eventos en un proceso.

Por estas razones Latour establece que la construcción de realidad actancial es plena en sentido fuerte: nada del actante es exterior a la performación que lo pone en el mundo, él, por tanto, carece de potencia. Sin embargo sería insuficiente, según Latour, considerar dicha realidad de manera aislada. Según su principio de realidad, ésta es compuesta por el actante con otros actantes (1984, pp. 185-6). Esta composición no sigue la lógica del reconocimiento, sino la del tamaño alcanzado por la movilización. Las variaciones de tamaño (o "cadena de traducciones") del actante generadas por su movilización (alianzas con otros actantes) aumentan o disminuyen su realidad. Sin dejar de ser plena, la realidad actancial no es un dato constante, sino un dato variable dependiente del tamaño. Dentro de este marco la ontología de la ANT establece las propiedades básicas de los actantes: traducción, unidad abstracta e irreductibilidad.

- Traducción: este es el concepto central de la ontología relacional de la ANT.16 La traducción es una operación, la única capaz de establecer una relación entre unidades irreductibles y heterogéneas (actantes). Para ello la traducción construye un medio, y en él establece una relación no-inmediata entre aquéllas, es decir, media la composición de los actantes sin comunicarlos. Traducción significa así "creación de un lazo (link) que no existía antes, que, hasta cierto punto, modifica dos elementos o agentes" (1994, p. 32) La traducción supone heterogeneidad, y además la regenera y multiplica. En la operación de traducción, la heterogeneidad de los actantes y el gradiente de sus fuerzas se inestabiliza. En virtud de ello, y sólo de ello, la traducción es fuente tanto de fuerza (source de la force) como de propiedades actanciales, que únicamente así moviliza realidad en el mundo. Sobre esta base es planteado el relacionismo de la heterogeneidad. Este concepto cobra toda su dimensión a partir del principio de irreductibilidad, pues no puede suponer la anterioridad de los relata sobre la relatio ni predicar prius en la misma.

- Unidad abstracta: los actantes carecen de cualidades y propiedades inherentes. Las cualidades y propiedades actanciales emergen de la operación de traducción que los performa dentro del mundo y se alojan en la unidad eventual del actante, no en sus componentes aislados o agregados. La traducción impone a los actantes una unidad abstracta, que no responde ni al concepto de unidad simple ni al de unidad

\footnotetext{
16 Este concepto fue elaborado originalmente por Michel Serres (1974). Callon (1986) lo retomó tempranamente y Latour lo prosiguió.
} 
holista, sino al concepto de entelequia, es decir, una unidad heterogénea de componentes heterogéneos (1984, p. 178). ${ }^{17}$

- Irreductibilidad: es el primer principio de la ANT y pregona que ninguna cosa es por sí misma reductible ni irreductible a ninguna otra (1984, p. 177). Ello funda la heterogeneidad radical entre los actantes y acentúa que la emergencia de la unidad abstracta del actante no es causada por, ni es un epifenómeno de, principios eficientes primeros y exteriores. Además, en tanto irreductibles, los actantes son inequivalentes pues, ante la falta de medidas universales subyacentes, ninguna unidad emergente es por sí misma igual o diferente a otra (1984, p. 181).

Sobre esta base la ANT planteó una ontología actualista y simétrica, que sustituye al sustancialismo, al esencialismo y al sujeto.

- Actualismo: la ANT optó por una perspectiva ontológica actualista y criticó al sustancialismo por la insuficiencia de la categoría de sustancia para describir la plenitud del actante. ${ }^{18}$ Esto surge de la composición de la unidad abstracta de un actante depende plenamente de la traducción y las traducciones son mediaciones contingentes de determinadas propiedades (fuerza, materia, alianza, espacio y lugar), por consiguiente, en la medida en que la traducción determina la composición actancial, la sustancia una y necesaria le es exterior al actante, que es por definición insustancial.

- Simetría: Latour criticó la asimetría ontológica y epistemológica del dualismo sujeto/objeto. Según el autor la ontología actancial fundada en la traducción es refractaria al dualismo: la traducción compone actantes heterogéneos en materia y fuerza de manera tal que el principio activo es ejercido por una unidad actancial de quasi-sujetos y quasi-objetos, en el sentido de Serres. Sobre esta base cuestionó la separación ontológica entre un polo absolutamente activo ('el sujeto') y otro polo meramente pasivo ('el objeto'). Basada en el principio de la traducción, la simetría no significa desdiferenciación ontológica, sino impertinencia de la asimetría para tratar la heterogeneidad.

\section{TAC: constitución lingüística y concepto formal de mundo ${ }^{19}$}

Habermas cuestionó las diversas bases y tradiciones filosóficas de la "metafísica occidental". En contra de la cual elaboró un componente ontológico postmetafí-

17 Harman (2009, p. 106) criticó la tesis de Latour sobre la componibilidad del actante, porque carecería de unidad por regresión al infinito, en modo análogo al nominalismo. Consideramos inapropiada dicha crítica puesto que Latour conecta su eventualismo (Harman lo encuadra en el ocasionalismo) con el concepto de asociación, dota así de unidad temporal y acontecimental al actante y evita la regresión al infinito.

18 Harman (2009, pp. 127-30) estableció la filiación de la metafísica de Latour con el actualismo.

19 En cuanto a la Teoría de la Acción Comunicativa, la producción teórica de Habermas se extiende a lo largo de más de cinco décadas, en ella distinguimos tres fases: la primera (1955-1971) se caracteri- 
sico centrado en un concepto racionalista y fáctico del lenguaje, conectado con una perspectiva evolutiva de las competencias universales resultantes del proceso de hominización, a las que consideró alojadas en las estructuras profundas del género humano. Habermas planteó un giro lingüístico universalista y racionalista contrario al perspectivismo y al idealismo, fuertemente orientado a la facticidad mundana, en conexión con un materialismo histórico reconstruido, tan contrario al empirismo como al trascendentalismo. Sobre esta base la TAC rechazó los principios fuertes de la metafísica sustancialista, idealista o realista, y se situó entremedio del "idealismo lingüístico" y del "realismo metafísico".

La tesis ontológica básica de Habermas es que el lenguaje constituye el acceso al mundo de los hombres. En ella concurren tres afirmaciones fundamentales: primero, en términos de un materialismo histórico reconstruido en conexión con la antropología de la hominización, el lenguaje es una adquisición evolutiva intramundana; segundo, en términos de un racionalismo transformado, el lenguaje constituye el sentido de los hombres, y tercero, en términos de la teleología de una pragmática universalista, el lenguaje es el medio del entendimiento entre los hombres. En torno a ellas, sin necesidad de una base cosmológica mayor, Habermas articuló un constitucionalismo lingüístico fuerte con un realismo no-metafísico. ${ }^{20}$

\footnotetext{
za por el desarrollo de una teoría de la praxis, coronada en la elaboración de la teoría del interés emancipatorio; el punto de inflexión es la publicación de Zur Logik der Sozialwissenschaften ("La lógica de las ciencias sociales"). La segunda fase (1971-1997) se caracteriza por la asunción de un "giro hacia el entendimiento" y sobre la elaboración de una teoría de la comunicación comunicativa. En ella podemos distinguir dos subfases, en la primera (1971-1981) delinea el programa de una teoría de la acción en conexión con la pragmática universal, el punto de inflexión es la publicación de Theorie des kommunikativen Handelns ("Teoría de la acción comunicativa"); la segunda subfase (1981-1997) fundamenta la teoría de la acción comunicativa y desarrollada una teoría de la sociedad y de la racionalidad sobre su base, la inflexión es la publicación de Faktizität und Geltung ("Facticidad y validez"). El tercero (1997 hasta la actualidad) se caracteriza por la reformulación parcial de los fundamentos y del programa práctico de la TAC. Aquí enfocaremos la segunda fase, en ese momento Habermas imprimió a la TAC los giros lingüístico y pragmático, así diferenció su fundamento operativo del "paradigma funcionalista" y del "paradigma de la acción", a los que contrapuso la acción comunicativa; se diferenció además del programa de la Teoría Crítica, al que contrapuso la racionalidad comunicativa. En ese marco, Habermas impulsó un programa orientado al entendimiento, con pretensión de universalidad y actitud performativa. Concibió la acción comunicativa como fundamento operativo novedoso y en torno a ella planteó un componente ontológico basado en la constitución lingüística, un componente epistemológico sostenido en la pragmática formal, y un componente metodológico orientado a la reconstrucción racional.

20 Lafont (1993) y Fabra (2008) coinciden en que con el concepto formal de mundo adviene a la TAC una intuición realista. En esta línea, la tensión entre constitucionalismo lingüístico y objetividad del mundo compartido es propia de la TAC. Fabra (2008, p. 287) agregó que Habermas concibe la objetividad sin premisas realistas fuertes como la suposición de un mundo objetivo externo e independiente de nosotros.
} 
- El universalismo es intramundano: apoyado en la antropología del proceso de hominización Habermas asume que el lenguaje no es un dato necesario del mundo, sino una adquisición evolutiva del género (Gattung) homo sapiens sapiens. El lenguaje, la cognición y la interacción son competencias formadas de un modo absolutamente intramundano mediante variaciones ontogenéticas y gradual transmisión génica de mutaciones a lo largo del proceso de hominización. Como tales tienen una base estrictamente biológica cuyos basamento orgánico y transmisión filogenética se estabilizaron 40.000 años atrás. Desde entonces la evolución del hombre fue únicamente socio-cultural, y ya no biológica ni biológica-cultural. Concebido en estos términos el lenguaje es una de las tres competencias universales de los hombres. En cuanto tal es una estructura profunda del hombre, es decir, una competencia filogenéticamente humana, debido a que es una condición de posibilidad para que cada individuo de la especie la desarrolle y sea capaz de performar acciones a partir de ella, al igual que sus congéneres. ${ }^{21}$ Debido a ello, el lenguaje es una región del mundo, plenamente intramundano, tiene lugar dentro del mundo material y no fuera de él, no constituye instancia ni posee propiedad de naturaleza extramundana. Por esta razón es plenamente fáctico en su potencialidad y en su actualización. Es importante señalar que, según Habermas, las otras dos estructuras profundas (la competencia cognitiva de la acción instrumental y la competencia interactiva de la socialización) son, al igual que la competencia lingüística, específicas y autónomas, sin embargo al organizarse lingüísticamente ambas, ofrecen un acceso a ellas desde el lenguaje y una preeminencia de éste sobre ellas.

- Concepto formal de mundo: Habermas justificó la introducción del concepto de mundo dentro del marco de la constitución lingüística del sentido debido a que "los propios sujetos socializados, cuando participan en procesos cooperativos de interpretación, hacen un uso implícito del concepto de mundo" (1981-I, p. 123). Este concepto de mundo es una unidad plena de sentido con rango objetivo, cuyo uso referencial no es individual, ni esporádico ni tampoco aislado, sino intersubjetivo y permanente, pues es común a todos los sujetos y es compartido por todos ellos como tal. En esta medida el concepto de mundo cumple una función especial: establece un correlato compartido y común de las interacciones lingüísticamente mediadas. En otras palabras, para la aceptación o rechazo de las pretensiones de

\footnotetext{
${ }^{21}$ La distinción entre competence y performance sigue la lógica del planteo de Chomsky, pero lo reformula parcialmente al reemplazar la fundación de Chomsky de las competences sobre la base del cogito cartesiano por una base evolutiva. Apel (1985 T2, p. 279 n. 72) objetó el concepto habermasiano de competence por incurrir en monologismo. Si bien la objeción es aguda, es impertinente por cuanto la base del concepto de competence no es un solus ipse sino una propiedad genérica, filogenéticamente universal a todos y cada uno de los individuos "normales". Habermas describe su desarrollo ontogenético como aprendizaje individual, pero manifiesta que esa descripción es general por cuanto sucede simultáneamente en otros congéneres. Por consiguiente, la competence es filogenética, pero su desarrollo y su performance, ontogenéticos.
} 
validez establece dentro del lenguaje una medida plena de sentido y común a los sujetos, pero no sujeta de ellos. Es decir, sin renegar de la constitución lingüística en sentido fuerte, la TAC asume también que dentro del lenguaje el mundo por él abierto está ahí para todos, de manera que establece el soporte objetivo y común de los correlatos. Habermas asumió así que este concepto de mundo no designa "contenidos" sino únicamente la actitud de referir al mundo desde el lenguaje. En esa medida es un concepto formal de mundo y ofrece un correlato general para la identidad de significado de las reglas. Dichas pretensiones son juzgadas por hablante y oyente a partir del mundo de la vida. Éste no es una totalidad homogénea, sino que incluye co-originariamente tres mundos plenos de sentido: el mundo de los hechos (mundo objetivo), el mundo de las normas (mundo intersubjetivo) y el mundo interior de vivencias (mundo subjetivo) (1981-I, p. 125-6). La común orientación hacia este mundo determina que toda pretensión de validez es susceptible de verdad y crítica.

- Orientación teleológica: según Habermas el entendimiento es el telos inmanente del lenguaje, aquél habita en éste y ambos conceptos se interpretan, así, recíprocamente (1981-I, p. 387). Debido a ello predomina en él la función comunicativa, ésta consiste en el uso de un lenguaje proposicionalmente articulado por parte de sujetos capaces de habla y acción con el fin de alcanzar el entendimiento y coordinar planes de acción. En este sentido el lenguaje consiste en fuerzas ilocucionarias. Ellas habitan dentro (innenwohnen) del lenguaje y están distribuidas en él como posibilidades. Los sujetos capaces de lenguaje son capaces de manejar las reglas lingüísticas de modo tal que actualizan dichas posibilidades intersubjetivas en situaciones de habla con el fin de alcanzar el entendimiento y el acuerdo entre ellos (1984, pp. 84-5). Dicha puesta en acto de las fuerzas ilocucionarias, y sólo ella, genera un efecto de vínculo (Bindungseffekt) entre los hombres. Por consiguiente, la generación de entendimiento intersubjetivo, tanto el telos inherente al lenguaje en su uso comunicativo como el fin que puede alcanzarse o no alcanzarse, nada tiene de necesario ni de natural. Es decir que la intersubjetividad del lenguaje natural existe porque, en principio, es posible el acuerdo, no porque sea menester empezar a entenderse.

La ontología de Habermas se completa con la definición situada de racionalidad, que el autor estimó alcanzar al conectarla con los planteos precedentes. Lo dicho, racionalidad en Habermas significa capacidad para la adquisición de conocimiento falible. En la medida en que está fundada en una competence universal (la capacidad de adquisición) y consiste en conocimiento falible, la racionalidad es universal, pero no trascendental. Está sujeta al desarrollo de capacidades y a la evolución del conocimiento. Habermas infiere que el aprendizaje y los contenidos dependen originalmente de la evolución del entendimiento (cognitivismo y falibilismo), por lo que la racionalidad depende de éste y queda con él situada dentro del mundo 
y su propia evolución. Es decir, la racionalización del mundo de la vida únicamente puede tener lugar de manera intramundana y estar mediada por el uso comunicativo del lenguaje orientado al entendimiento. Por todas estas razones Habermas postuló al nivel ontológico una conexión sistemática entre racionalidad y entendimiento, cuyo apego a las reglas del procedimiento de adquisición y evolución, no obstante su acento sobre la mundanidad y su carácter situado, le valió ser calificada como racionalidad procedimental. Las racionalidades de la acción teleológica (racionalidad instrumental) y la acción estratégica (racionalidad estratégica) conservan su autonomía, pero están organizadas por tal conexión sistemática.

\section{Comparación}

\subsection{Tiempo y espacio}

\section{Convergencias}

Los tres programas distinguen los conceptos de Tiempo y Espacio de los conceptos de temporalidad y espacialidad. En el primer par (Tiempo, Espacio) predomina la concepción física del mundo y sus propiedades físicas son fundamentales respecto de las propiedades de las entidades. Gracias a ello ambos conceptos son definidos como dimensiones y condiciones fundamentales para la formación y composición de entidades. En cambio, en el segundo par (temporalidad, espacialidad), los tres programas desdoblan conceptualmente al Tiempo y al Espacio y los ponen en relación con las entidades, no sólo como dimensiones físicas fundamentales, sino como condiciones bajo la cuales las entidades definen de manera relativa la cualidad de su situación dentro del mundo. Gracias a ello, la temporalidad y la espacialidad tienen el curioso rango de conceptos generales y derivados, que designan propiedades relativas y variables de entidades situadas bajo condiciones fundamentales.

En relación con el Tiempo, en los tres casos se observa, en primer lugar, una definición física del Tiempo como continuo sincrónico fundamental. Más allá de algunas menciones aisladas de Luhmann y Latour a la física de la reversibilidad de Prigogine y Stengers y a los "avances de la física cuántica", ninguno problematizó especialmente la definición física newtoniana-einsteniana del tiempo, la que simplemente asumieron. En segundo lugar, los tres distinguen Tiempo y temporalidad, pero desarrollan sus planteos ontológicos a partir del segundo, pues, de ambos, es el único relativo a las entidades y los fenómenos. Los tres autores conciben la temporalidad como heterónoma y sincrónica respecto del Tiempo del mundo, con propiedades específicas como la discontinuidad y la procesalidad. Para los tres autores la temporalidad es heterónoma porque la temporalidad dura con el Tiempo del 
mundo y lo supone, y es sincrónica porque no hay temporalidad fuera del tiempo, sucede dentro del Tiempo y de manera simultánea. Es discontinua porque la temporalidad está constituida por unidades discretas de duración relativa denominadas acontecimientos, que constituyen puntos irreversibles de la temporalidad. Por ello los acontecimientos puntualizan y discontinúan la temporalidad dentro de la continuidad del tiempo, dado que en relación con cada punto hay acontecimientos anteriores y posteriores, es decir un antes y un después. Es procesal porque genera secuencias mediante la vinculación longitudinal de acontecimientos diversos.

En relación con la definición ontológica de acontecimiento, los tres programas tienen una convergencia aún más importante que las ya analizadas, a saber, los tres estrechan la definición de sus unidades de análisis con el concepto y la propiedad ontológicos de acontecimiento o evento. Esto se observa en los conceptos de operación de Luhmann, puesta en acto de Latour y acto de Habermas. Los tres conceptos definen la unidad de análisis como acontecimiento: la ciñen a una unidad temporal, la dotan de duración e irreversibilidad y la puntualizan dentro de la temporalidad. Por esta razón, el concepto de acontecimiento es central en la ontología de estos programas de las entidades, las propiedades y las condiciones dentro del mundo. Debido a sus dialogos con el eventualismo de Whitehead, esta plataforma es más fuerte en Luhmann y Latour que en Habermas, pero no está ausente en el concepto de acto de éste, de manera que la diferencia de acentos no anula la convergencia.

En relación con el Espacio, en los tres casos se observa, en primer lugar, una definición física del Espacio como extensión simultánea fundamental. Ninguno problematizó especialmente la definición física newtoniana-einsteniana del espacio, la que simplemente asumieron. En segundo lugar, de modo análogo al desdoblamiento entre Tiempo y temporalidad, los tres distinguen Espacio y espacialidad y desarrollan sus planteos ontológicos a partir del segundo, ya que es el único de ambos relativo a las entidades y los fenómenos. Los tres autores consideran que la espacialidad es heterónoma respecto del Espacio del mundo y posee propiedades específicas como la distancia. Es heterónoma porque la espacialidad supone al Espacio, tiene lugar dentro de él y lo supone. Posee la propiedad de la distancia porque puntualiza la extensión y con reglas propias mensura los segmentos comprendidos, establece así diferencias entre distancias mayores y menores dentro del Espacio. En este aspecto hay una convergencia problemática entre los autores. A los tres se les ha cuestionado que reducen la espacialidad a la distancia, debido a que sólo la conciben en función del Tiempo.

\section{Divergencias}

No hallamos divergencias de fondo entre estas concepciones del Espacio y el Tiempo. Identificamos solo dos divergencias de acentos, a saber: 1) el carácter pro- 
blemático del acontecimiento para las entidades y 2) la pluralidad de temporalidades. La primera es la más destacada y se observa en los conceptos de "coacción a seleccionar" (Selektionszwang) de Luhmann y de "evanescencia" de Latour, ellos remarcan el carácter problemático de la temporalidad del acontecimiento para las entidades, pero sobre todo enfatizan el carácter constitutivo que tiene para ellas. Habermas, en cambio, aloja los acontecimientos en la unidad temporal de los actos que tienen lugar en las situaciones de habla, pero no los considera problemáticos ni constitutivos desde la perspectiva de los actores.

La segunda divergencia se vincula con el interés de Latour por la heterogeneidad entre fuerzas y materias del mundo, interés que lo llevó a distinguir la pluralidad heterogénea de temporalidades no-isocrónicas que se pliegan en la temporalidad de cada acontecimiento. La distinción directamente está ausente en Luhmann y Habermas. Cabe conectar esta divergencia con los antecedentes filosóficos, por un lado, Luhmann y Habermas retoman de sus diálogos con la fenomenología la concepción de la unidad temporal de las temporalidades relativas. Latour, en cambio, antepuso su concepto de irreductibilidad y cuestionó la idea y el alcance de una metaunidad temporal que fusione las temporalidades no-isocrónicas, se apoyó para ello en el concepto de pliegue de Deleuze (Farías 2014).

\subsection{Composición de entidades}

\section{Convergencias}

En esta dimensión las convergencias entre la TGSS, la ANT y la TAC son relevantes y numerosas. La primera de ellas es la concepción mundana: La complejidad de la TGSS, la heterogeneidad de la ANT y el concepto formal de mundo de la TAC conciben los fenómenos, las entidades y sus condiciones de posibilidad de manera plenamente mundana. En los tres casos el mundo donde lo social acontece es el único mundo posible y como tal es intranspasable. En torno a ello se nuclean otros cuatro puntos en común, primero, los tres adoptan una perspectiva inmanentista y critican la perspectiva trascendentalista; segundo, los tres aceptan que dentro de ese único mundo existen fuerzas fundamentales, cuya descripción conceden a la concepción física del mundo; tercero, y no obstante dicha concesión, los tres rechazan la postura fisicalista, pues aquélla no fuerza la asunción ni de explicaciones ni de reducciones causales; cuarto, los tres conciben la cualidad de modo intramundano, así la cualidad no niega la descripción física, pero tampoco se deja reducir a ella.

La segunda convergencia es la definición del principio composicional como cualificación. Para los tres programas la composición de entidades, tanto su emergencia como su reproducción, es una formación de cualidades que sucede dentro del mundo con precursores y condiciones igualmente mundanos. La cualidad, por esta razón, no es ni un accidente ni un atributo, sino una propiedad fundamental y dis- 
tintiva de las entidades, que las diferencian de manera necesaria y suficiente. En esta definición el concepto de acontecimiento juega un papel primordial, con ello la convergencia gana profundidad, ya que para los tres autores la composición es un acontecimiento de cualificación. Ninguno de ellos acepta que las cualidades estén dadas necesariamente en el mundo. Para ellos las cualidades se componen de un modo y en un momento específico en relación con determinadas entidades, y no con otras, más aún, para los tres las cualidades duran con las entidades y están sujetas a variación. En este punto hay una convergencia muy importante en cuanto al fortalecimiento de la tesis de la cualificación, a saber, los tres autores declararon la irreductibilidad de las cualidades. Los tres autores plantean la irreductibilidad de las entidades a sus condiciones de posibilidad, en virtud de las propiedades unitarias de sus cualidades. El irreductibilismo ya no sólo es un principio negativo opuesto al fisicalismo, sino una propiedad positiva de la cualidad.

La profundidad de esta convergencia prolonga el planteo de la insustancialidad ontológica de las entidades formadas de manera múltiple hasta su reproducción y su extinción. Esto se observa en los conceptos de autopoiesis de Luhmann, inestabilidad de Latour y riesgos de la identidad de Habermas. Según estos conceptos las entidades persisten en el mundo mediante el encadenamiento continuo y forzado de unidades acontecimentales discontinuas. En los tres casos la entidad se extingue si el encadenamiento cesa. Los conceptos describen así la dependencia ontológica de las entidades respecto de los acontecimientos y declaran la plena facticidad de las entidades. De esta manera los planteos desembocan, finalmente, en que los tres programas afirman fuertemente la facticidad de las cualidades y de las entidades, como se observa en las tesis de "hay sistemas" en Luhmann, "los colectivos están en el mundo" de Latour y "la acción es fáctica y la racionalización lo es con ella" de Habermas. 22

La tercera convergencia es la crítica al elementalismo y la opción por la formación múltiple. Los tres programas rechazan la tesis del elementalismo según la cual hay entidades simples en el mundo, capaces de ser agregadas en unidades mayores homogéneas. En contra de ella asumen que las entidades se componen mediante formación múltiple, es decir, mediante la interacción entre acontecimientos heterogéneos en un momento dado. Esto se observa en los conceptos de emergencia de la TGSS, performación de la ANT y generación de la TAC que designan la aparición cualitativamente novedosa de las entidades en el mundo, cuya composición nunca es cualitativamente simple, sino cualitativamente acontecimiental. Descontadas las divergencias sobre el tipo de entidad formada, los tres conceptos asumen que nin-

\footnotetext{
22 Descartamos la crítica de Habermas contra Luhmann respecto de la ausencia de un concepto de "muerte" de sistemas. Para la TGSS los sistemas complejos se extinguen, la "muerte" es la forma específica de extinción de los sistemas "vivos". Los sistemas psíquicos y sociales no viven, por ende, su forma de extinción no podría ser la muerte, sino se estaría ante una analogía biologicista.
} 
guna entidad está dada en el mundo, ellas aparecen como formaciones múltiples de unidades cualitativas, están ligadas temporalmente al acontecimiento y su composición no se reduce ni a las condiciones de posibilidad ni a las cualidades de los componentes o precursores. Latour y Luhmann retomaron el concepto de traducción de Serres. Habermas también avanzó en esa línea, pero por la vía de la distinción entre competence y performance.

Esta convergencia tiene un grado más de profundidad. Para los tres autores la tesis de la formación múltiple muestra la precariedad de la unidad cualitativa de las entidades y su fuerte dependencia respecto de las condiciones del mundo. A causa de ello las entidades son, en sentido fuerte, incapaces de fundamentarse a sí mismas y aún más incapaces de fundamentar al mundo. Los autores infirieron que ninguna de ellas es un subiectus en sentido filosófico estricto y rechazaron toda concepción subjetual.

\section{Divergencias}

En esta dimensión hallamos tres divergencias significativas. La primera de ellas es el modo de composición de entidades. Luhmann propone un concepto de síntesis, Latour, un concepto de hibridación performativa y Habermas, un concepto de puesta en acto. En los tres autores hay irreductibilidad y cualidad, pero Luhmann propone una unidad sintética, Latour, una unidad heteróclita y Habermas, una unidad racional. Para identificar la sutileza de esta divergencia acudimos a la distinción emergentista entre integral y modular (Bunge 2004, pp. 26-9). La síntesis concebida por Luhmann es congruente con una composición integral, mientras la hibridación de Latour y la performance de Habermas son congruentes con una concepción modular. Esta divergencia es extensiva a los principios composicionales de cada programa: la emergencia de la TGSS es integral, mientras que la performación de la ANT y la generación de la TAC son modulares. Por ello, las posiciones de los autores divergen modalmente, aunque converjan conceptualmente.

La segunda divergencia tiene que ver con un aspecto poco atendido hasta el momento: la amplitud o la estrechez del encuadre inicial. Luhmann y Latour parten de premisas cosmológicas, Habermas lo hace, en cambio, desde el proceso de hominización. Las pretensiones de cada programa juegan un papel, de hecho la pretensión de universalidad de la TAC explica la divergencia. En el encuadre ontológico inicial de la TAC no hay premisas cosmológicas porque no son necesarias para establecer las estructuras de la racionalidad, basta al efecto partir del proceso de hominización. Ni la pretensión de universalidad de la TGSS ni la pretensión de simetría generalizada de la ANT se concentran en la racionalidad, ni siquiera en el "hombre". Están obligados a partir de otro encuadre. La pretensión de universalidad de la TGSS se concentra en la operación de sistemas complejos, para cuyo abordaje cabe exigirle la inclusión de los rudimentos de una cosmología de la complejidad. 
Sin ellos carecería de una base adecuada. La ANT, en tanto, estableció su generalización en torno a la simetría de fuerzas y materias, y la encuadró en una teoría de la heterogeneidad y la traducción, por lo que cabe exigirle los rudimentos de una cosmología de la hibridación. Sin ellos también se desmoronaría. Ambos programas están obligados a incluir en sus componentes ontológicos premisas cosmológicas (físicas, químicas, materiales, biológicas). De este modo logran un encuadre inicial más amplio que la TAC. ${ }^{23}$ Cabe señalar que estos encuadres no hacen concesiones fisicalistas, materialistas o biologicistas, pues sólo encuadran las cualidades, no las reducen.

\subsection{Relaciones causales entre entidades}

\section{Convergencias}

En esta dimensión hallamos dos convergencias significativas. La primera de ellas es el rechazo del causalismo y la opción por modelos cualitativos. Para establecerla sin apresurar conclusiones, apelamos a la distinción entre relaciones causales y explicaciones causales (García 2006, p. 78). Así vistos los tres autores proceden de igual modo: primero, aceptan un modelo restringido de relaciones causales al nivel de las condiciones de posibilidad; segundo, rechazan un modelo ampliado al nivel de las cualidades, en reemplazo del cual elaboran modelos relacionales específicos, y tercero, rechazan de plano las explicaciones causales al nivel epistemológico. Ampliemos el punto. Los tres programas adoptan el modelo de relación causal restringida y aceptan en consecuencia que las entidades (sistemas, colectivos, plexos) cumplen con las legalidades del mundo, pues no crean el mundo, sino que lo presuponen. Esta "cobertura nomológica" de las condiciones de posibilidad (la apertura causal del sistema al entorno en la TGSS, la objetividad de la heterogeneidad de la ANT, la dimensión física de los sistemas de acción en la TAC) es congruente con la tesis de la mundanidad. Sin embargo, los tres programas restringen el alcance del modelo y declaran su impertinencia ante la relación cualitativa específica. Así ni la cualidad de las entidades ni sus relaciones se deducen de las relaciones condicionales (tal deducción incurriría en fisicalismo). Para este abordaje los tres autores proponen modelos relacionales cualitativamente específicos atentos a su composición y su facticidad. Gracias a este proceder, el modelo de relación cau-

\footnotetext{
23 En su cotejo de Bourdieu, Habermas y Giddens, Scribano (2009, p. 203) concluyó acertadamente que la centralidad de la razón es un factor de divergencias entre dichos autores. Esta conclusión le cabe también a nuestro corpus, aunque por otras causas, en aquel caso la centralidad de la razón ampliaba el componente ontológico de la TAC respecto de los planteos de Giddens y Bourdieu, pero en relación con los planteos de Luhmann y Latour lo reduce, pues "sólo" parte del proceso de hominización para llegar a las estructuras profundas de la racionalidad, limita así el acceso de la TAC a la discusión cosmológica y a la exploración interdisciplinaria de la estructuración de lo real.
} 
sal restringido se muestra compatible con un modelo cualitativo y relacional específico (emergencia y diferenciación en la TGSS, actante y traducción en la ANT, entendimiento y acuerdo en la TAC). Los conceptos cualidad y causalidad ocupan así el centro del componente ontológico de la TGSS, la ANT y la TAC. Sobre esta base relegaron epistemológicamente la explicación causal.

La segunda convergencia es el rechazo del modelo relacional todo/partes y la opción por modelos contingentes. Los tres programas objetaron el modelo todo/parte debido a sus insuficiencias para tratar la autonomía relativa y las diferencias de nivel de las entidades. La ANT y la TAC lo trataron como un modelo holista y le objetaron la reducción de las relaciones de las entidades a su funcionalidad. La TGSS amplió dicha crítica: no sólo es reduccionista, incurre además en indeterminación, pues, si la identidad del todo determina la identidad de la parte y la identidad de las partes determinan la identidad del todo, hay un regreso al infinito entre ambos niveles sin que alcancen la determinación. Consumado el rechazo, los tres programas optaron por modelos relacionales contingentes. Como se observa en los conceptos de selección de la TGSS, mediación de la ANT y acción de la TAC, en estos modelos toda relatio establece entre los relata vínculos siempre posibles de otro modo, es decir vínculos ni necesarios ni imposibles, basados en la contingencia de la relación. Los relata según nuestros autores pueden ser elementos o estructuras, por tanto la relación entre unidad acontecimental y formación estructural también se ciñe a este modelo contingente, como vemos en la relación entre comunicación y expectativa en la TGSS, asociación y colectivo en la ANT y acción y plexo en la TAC.

\section{Divergencias}

En esta dimensión hallamos una divergencia significativa. Se trata de la especificidad de la cualidad según se base en el principio de identidad (relaciones de significado y validez en la TAC) o en el principio de la diferencia (relaciones autorreferenciales en la TGSS, relaciones de traducción en la ANT). En el primer caso la cualidad es desplegada como un principio identitario en un ámbito homogéneo, el principio es común y general y es capaz de distinguir y relacionar unidades. En el segundo caso las relaciones son inequivalentemente cualificadas y la cualidad es desplegada como un principio de diferencia en un ámbito heterogéneo y el principio es capaz de distinguir y relacionar unidades. Destacamos, primero, que existe una fuerte convergencia entre la TGSS y la ANT respecto del principio de la diferencia, calificada como "interfase" por Farías y Ossandón (2006), ello los diferencia de la TAC: el modelo relacional de la TAC es para ellos insuficiente pues se limita a ampliar el análisis de las condiciones, mientras los modelos relacionales de la TGSS y de la ANT son insuficientes para la TAC, ya que no fundamentan la base común de ella; segundo, la elección de un principio u otro, sin embargo, no afecta 
la consecución ni de la distinción ni de la relación.

Esta interfase entre TGSS y ANT merece una ampliación. La diferencia sistema/entorno y sistema/sistema en la TGSS y el principio de traducción de la ANT comparten el principio de la diferencia para basar y modelar el planteo relacional entre entidades. Esto significa que entre las cualidades no hay relaciones de correspondencia, de identidad o de necesidad, sino diferencias que remiten a diferencias. Por lo tanto, en ambos casos las relaciones son alojadas en el campo de la no-linealidad. La TGSS acentúa la producción de información que genera la diferencia y eleva ese elemento al rango de principio relacional que rige tanto la orientación del sistema hacia el entorno (relaciones de gradiente e incertidumbre), como hacia los sistemas en el entorno (relaciones intersistémicas), incluso al interior de los sistemas (relación de autoobservación). La ANT acentúa la formación de intereses mediante la traducción de diferencias, al interior incluso de los colectivos. Esta convergencia se vincula con la convergencia en el antecedente de Michel Serres. ${ }^{24}$

\section{Conclusiones: ontologías débiles con facticidad fuerte}

A lo largo de la exposición cotejamos los componentes ontológicos de Luhmann, Latour y Habermas. En todos los ejes de análisis hallamos convergencias y divergencias, aunque varían en número y fuerza según el eje. En algunos casos sin embargo logramos agrupar pares de posiciones. El eje "composición de entidades" muestra una alta frecuencia de convergencias, mientras que en los ejes "tiempo y espacio" y "relaciones causales" convergencias y divergencias se equiparan, aunque las primeras tienen un valor especial porque contradicen las recepciones recíprocas y los estándares interpretativos que reducen la comparación entre los autores a unas pocas divergencias. Ordenamos los resultados en la tabla que sigue a continuación.

\footnotetext{
${ }^{24} \mathrm{La}$ tesis sobre la incomunicabilidad de sustancias de Serres nos ayuda a entender la apoyatura que en él hallaron Latour y Luhmann. Según Serres, Kant resolvió de modo incorrecto la segunda antinomia debido a que la comunicación entre entidades no existe, por lo que es necesario retrotraer la discusión sobre la composición de entidades a la alternativa de Leibniz frente al dilema realismo/nominalismo.
} 
Tabla. Convergencias y divergencias entre componentes ontológicos

\begin{tabular}{|c|c|c|}
\hline Tiempo & Convergencias & Divergencias \\
\hline Espacio & $\begin{array}{c}\text { Distinción Tiempo/temporalidad } \\
\text { Distinción Espaciolespacialidad } \\
\text { Definición física de Tempo Espacio } \\
\text { Relatividad de espacialidad y temporalidad } \\
\text { Centralidad del acontecimiento }\end{array}$ & $\begin{array}{c}\text { Problemática del acontecimento } \\
\text { Pluralidad de temporalidades }\end{array}$ \\
\hline $\begin{array}{c}\text { Composición } \\
\text { de entidades }\end{array}$ & $\begin{array}{c}\text { Rechazo del sustancialismo, esencialismo } \\
\text { y trascendentalismo y opción intramundana } \\
\text { Composición cualificada, facticidad plena } \\
\text { e irreductibilidad de las entidades } \\
\text { Rechazo del elementalismo y opción } \\
\text { por la composición múltiple }\end{array}$ & $\begin{array}{c}\text { Modalidad de composición } \\
\text { Encuadre inicial }\end{array}$ \\
\hline $\begin{array}{c}\text { Relaciones } \\
\text { causales }\end{array}$ & $\begin{array}{c}\text { Rechazo del causalismo y opción cualitativa } \\
\text { Rechazo de la totalidad y opción contingencia }\end{array}$ & Identidad/diferencia \\
\hline
\end{tabular}

A juzgar por los resultados nuestra conclusión es que los componentes ontológicos de la TGSS, la ANT y la TAC guardan importantes puntos de similitud y algunos puntos de contraste, estos mayormente modales, en cada uno de los ejes de análisis. Las similitudes indican convergencias entre concepciones de la estructuración de lo real que se proponen reemplazar los principios ontológicos de la tradición metafísica con principios interdisciplinarios y un marcado interés por la facticidad. Mientras que el punto nodal de las divergencias es el encuadre inicial y la opción por los principios de la identidad o de la diferencia.

Vistos sistemáticamente estos componentes ontológicos comparten tres elementos. En primer lugar, los tres rechazaron férreamente los principios y las perspectivas ontológicas fuertes, provenientes de las diversas tradiciones de la filosofía y la metafísica del pensamiento occidental. En segundo lugar, los tres optaron por fundamentos ontológicos alternativos, de origen científico, caracterizados por establecer principios y perspectivas respecto de la estructuración de lo real débiles en relación con las tradiciones ontológicas occidentales. En tercer lugar, los tres mostraron un marcado interés por redefinir conceptualmente el acceso a la facticidad y coincidieron en la elección del camino para hacerlo: La despojaron de principios empiristas latos, pero, para no renunciar a su fortaleza, acentuaron su conexión con las categorías acontecimentales fuertes.

De esta terna de elementos se sigue que los tres programas elaboraron en paralelo, aunque de modo convergente, un mismo punto de partida: criticar a la "tradición" y elaborar una concepción de estructuración de lo real integral y radicalmente alternativa a ella. En este punto nodal de las convergencias se articulan las críticas al sustancialismo, esencialismo y causalismo con la opción por un inmanentis- 
mo mundano, una temporalidad acontecimental, la formación contingente de entidades en el mundo y la no-linealidad relacional entre fenómenos y entidades y condiciones de posibilidad. Articulamos también la fuerte facticidad asumida por ellos, la que basaron en la unidad acontecimental de las entidades, la irreductibilidad de las cualidades y la plenitud concreta de los fenómenos. Esta articulación sistemática evita una trampa de las recepciones recíprocas entre los autores, que consiste en acusar de "tradicional" a cualquier programa que emplee apenas el término 'ontología', que implicaría, en especial para Luhmann, una concesión inaceptable. Nuestra conclusión sistemática es bien diferente: los tres programas rechazan de manera convergente el por ellos denominado "proyecto ontológico de Occidente" y aceptan una estructuración de lo real ontológicamente débil y fácticamente fuerte.

Esta convergencia entre componentes ontológicos nos lleva a reflexionar y sopesar mejor las divergencias programáticas entre perspectivas y distinciones directrices. Sin menoscabo de las divergencias entre un punto de partida sistémico, uno de redes y otro orientado al entendimiento, las convergencias entre los componentes ontológicos de los tres programas demuestra que las elaboraciones conceptuales tienen una lógica teórica que no puede ser reducida a las tradiciones, ya que, a pesar de ellas, elaboraron, en paralelo, conceptos y puntos de partida convergentes. Por tanto, los puntos de partida ya no son simplemente definiciones programáticas excluyentes y/o incluyentes, sino elaboraciones conceptuales que entremezclan elementos de una y otra índole, incluso a nivel ontológico.

\section{Referencias bibliográficas}

Apel, K-O. (1985): La transformación de la filosofia, 2 Tomos, Madrid, Taurus. Archer, M. (1988): Culture and Agency: The Place of Culture, Cambridge University Press.

Bourdieu, P. (1985): Qué significa hablar, Madrid, Akal.

Buckley, W. (1973): La Sociología y la Teoría Moderna de los Sistemas, Buenos Aires, Amorrortu.

Bunge, M. (2004): Emergencia y convergencia, Barcelona, Gedisa.

CALlON, M. (1986): "Éléments pour une sociologie de la traduction: La domestication des coquilles Saint-Jacques et des marins-pêcheurs dans la baie de SaintBrieuc", L'Année sociologique (1940/1948-), Troisième série, 36, pp. 169-208. Clam, J. (2000): "System's Sole Constituent, the Operation Clarifying a Central Concept of Luhmannian Theory", Acta Sociológica, 43(1), pp. 63-79.

Derrida, J. (1967): De la grammatologie, Paris, Les ëditions de Minuit.

D'Espagnat, B. (2006): On Physics and Philosophy, Princeton University Press.

Elster, J. (1978): Logic and Society: Contradictions and Possible Worlds. New York: John Wiley \& Sons. 
FaBRA, P. (2008): Habermas: lenguaje, razón y verdad, Madrid, Marcial Pons.

FARÍAS, I. (2014): "Virtual attractors, actual assemblages", European Journal of Social Theory, 17(1), pp. 24-41.

FARÍAS, I. y J. OSSANDÓN (2006): "Recontextualizando Luhmann. Lineamientos para una lectura contemporánea", en Farías, I. y J. Ossandón (Comps.): Observando sistemas. Nuevas apropiaciones y usos de la teoría de Niklas Luhmann, Santiago, RIL, pp. 17-54.

GarcíA, R. (2006): Sistemas complejos. Conceptos, método y fundamentación epistemológica de la investigación interdisciplinaria, Buenos Aires, Gedisa.

Giddens, A. (1984): The Constitution of Society: Outline of the Theory of Structuration, Cambridge, Polity Press

HABERmas, J. (1981): Theorie des kommunikativen Handelns. 2 Bde, Frankfurt, Suhrkamp.

HABERMAS, J. (1984): Vorstudien und Ergänzungen zur Theorie des kommunikativen Handelns, Frankfurt, Suhrkamp.

HABermas, J. (1986): La reconstrucción del materialismo histórico, Madrid, Taurus.

Hagner, M. y E. Hörl (2008): Die Transformation des Humanen. Beiträge zur Kulturgeschichte der Kybernetik, Frankfurt, Suhrkamp.

Harman, G. (2009): Prince of Networks, Melbourne: re.press.

HeidegGer, M. (2006): Sein und Zeit, Tübingen, Niemeyer.

Høstaker, R. (2005): "Latour - Semiotics and Science Studies", Science Studies, 18(2), pp. 5-25.

LAFONT, C. (1993): La razón como lenguaje, Madrid, Visor.

Latour, B. (1984): Les microbes, guerre et paix, suivi de Irréductions, París, Métailié-La Découverte.

Latour, B. (1994): “On Technical Mediation - Philosophy, Sociology, Genealogy", Common Knowledge, 3, pp. 29-64.

Latour, B. (2005): Reassembling the Social, Oxford University Press.

LuKÁCs, G. (1923): Geschichte und Klassenbewußtsein, Berlín.

Luhmann, N. (1984): Soziale Systeme, Frankfurt: Suhrkamp.

Luhmann, N. (2009): Soziologische Aufklärung, Bd. 5, Wiesbaden, VS Verlag.

MASCAREÑO, A. (2008): "Acción, estructura y emergencia en la teoría sociológica", Revista de Sociología, 22, pp. 217-256.

Maturana, H. y Varela, F. (2003): El árbol del conocimiento. Buenos Aires: Lumen.

Mead, G. H. (1931): Mind, Slef and Society, Chicago, Chicago University Press.

Neumann, J. v. (1966): Theory of Self-Reproducing Automata. Urbana: University of Illinois Press.

Parsons, T. (1953): "Some Comments on the State of the General Theory of Action”, American Sociological Review, 18(6), pp. 618-631. 
Pignuoli OcAmpo, S. (2015): "La posición epistemológica del constructivismo simétrico de Bruno Latour", Cinta de Moebio, 52, pp. 91-103.

RASCH, W. (2012): "Luhmann's Ontology", Revue internationale de philosophie, 2012/1 n 259, pp. 85-104.

RorTy, R. (1979): Philosophy and the Mirror of Nature, Princeton University Press.

Rosa, N. (1991): Léxico de lingüistica y semiología, Buenos Aires, CEAL.

Rustemeyer, D. (2007): "Die Logik der Form und das Problem der Metaphysik", Soziale Systeme, 13(1+2), pp. 504-515.

SCRIBANO, A. (2009): Estudios sobre teoría social contemporánea: Bhaskar, Bourdieu, Giddens, Habermas y Melucci, Buenos Aires, Ciccus.

SCHAEFFER, J. (2009): El fin de la excepción humana, México, FCE.

SCHÜTZ, A. (2003): Strukturen der Lebenswelt, Konstanz, UVK-UTB.

SERres, M. (1974): La Traduction (Hermés. Vol. III), París, Minuit.

Simmel, G. (1908): Soziologie. Untersuchungen über die Formen der Vergesellschaftung, Verlag Duncker \& Humblot Berlin.

TARDE, G. (1895): La logique sociale, Paris, Alcan.

TönNIES, F. (1887): Gemeinschaft und Gesellschaft. Abhandlung des Communismus und des Socialismus als empirischer Culturformen. Berlin.

VANDENBerghe, F. (2007): "Une ontologie realiste pour la sociologie. Systeme, morphogenese et collectifs", Social Science Information, 46(3), pp. 487-542.

Sergio Pignuoli Ocampo

UBA-CONICET-IIGG

spignuoli@conicet.gov.ar 